\title{
A Topology Optimization Formulation Applied to Highly Flexible Structures
}

\author{
Cícero Ribeiro de Lima ${ }^{1}$, André da Costa Teves ${ }^{2}$, Emílio Carlos Nelli Silva ${ }^{2}$ \\ ${ }^{1} U F A B C$-Federal University of ABC, cicero.lima@ufabc.edu.br \\ ${ }^{2}$ Escola Politécnica of University of São Paulo, ecnsilva@usp.br
}

\begin{abstract}
Highly flexible structures (springs) can be found in many precise devices, such as small actuators of optical disc drive and mobile cameras. A good configuration design is crucial for correct operation of these devices. In this case, optimization techniques can be applied to design of these flexible structures, aiming to reduce development time and costs. Remarkably, the design of these structures turns out to be a challenge in topology optimization. Thus, in this work, a formulation for designing highly flexible structures by using topology optimization is investigated. The topology optimization problem is defined as minimization of the mean compliance subjected to material volume and perimeter constraints, combined with a projection technique. The material model is based on the traditional SIMP approach and the optimization problem is implemented by using COMSOL software and solved by using MMA algorithm. A well-known plane string case has been carried out to evaluate the potential of the proposed topology optimization formulation.
\end{abstract}

Keywords: highly flexible structures, topology optimization, spring design, perimeter control

\section{Introduction}

A flexible structure can also be found, for instance, in accelerometer sensors that have movable electrode (proof mass), which is suspended by one or more springs [1]. The mechanical sensitivity of these precise devices is directly related to flexibility of its structures, that is, the more flexible the spring configuration, the higher the sensitivity of the device. Thus, a good configuration design of the flexible structure (spring) is crucial for their correct operation.

In this case, optimization techniques are regularly applied to the design of these structures, aiming to reduce development time and costs. Most of the research in optimization for flexible structure design has been primarily focused on parametric analysis [2]. An alternative approach widely used for optimal design is the Topology Optimization Method (TOM).

Typically topology optimization applied to structural design aims to obtain low compliance structure, and few works have been published on topology optimization for high compliance or flexible structures, such as springs. The closer known formulation for design of springs using the TOM is the one well-established for design of compliant mechanisms [3], which are mechanical structures with no-moving parts. This mechanisms transfer an input force, applied at determined point, to output displacement produced at another different point of the domain. However, the formulations of compliant mechanisms cannot be applied to the problem considered in this work (spring design), because the points of the applied input load and the desired output displacement are coincident. Song et al. [4] propose a two-stage search method to improve the convergence and the probability of finding good solutions using topology optimization applied to a leaf spring design.

Naturally, the topology optimization method tries to generate thin regions to obtain structures with high flexibility, as illustrated in figure 1a. However, it is evident that the manufacturing of this result is usually unfeasible and it would be better to obtain results as illustrated in figure $1 \mathrm{~b}$. By considering the same material and dimensions for design domain of figure 1 , the topology optimization result shown in figure $1 \mathrm{~b}$ is generally simpler to be manufactured, and allows high flexibility. However, it represents a local minimum result very difficult to obtain by using the traditional formulations of TOM.

The main feature that is different between the results illustrated in figure 1 is the perimeter of both topologies, in which the total perimeter of result shown in figure $1 \mathrm{~b}$ is about $30 \%$ higher than the one shown in figure 1a. Based on this observation and taking into account that slender structures have higher flexibility, in this work a new formulation is proposed for the topology optimization of such flexible structures (springs). Here, the optimization problem is defined for minimizing the volume of material subject to a mean compliance and perimeter constraint, combined with the projection technique. The SIMP material model [5] is adopted, and the continuation method is applied to avoid undesirable local minimum [6]. The projection scheme proposed by Bourdin [7] has also been applied as filtering technique. 


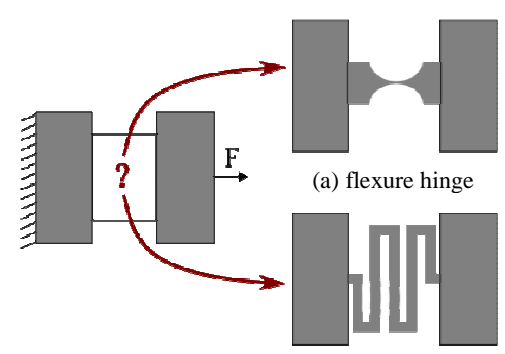

(b) zig-zag pattern

Figure 1: Structures with high flexibility: (a) thin structure configuration; (b) zig-zag configuration.

The topology optimization algorithm is implemented in MATLAB using the COMSOL software as the finite element solver. The potential of the proposed formulation is evaluated by designing a well-known plane string case.

This paper is organized as follows. In Section 2, the topology optimization formulation proposed for the design of flexible structures is described. Section 3 shows the sensitivity analysis, and Section 4 presents the numerical implementation of the topology optimization problem. The results are addressed in Section 5. Finally, Section 6 gives the concluding remarks and future developments.

\section{Proposed approach for topology optimization problem}

The classical formulation for structural optimization using the TOM is the mean compliance minimization [8]. In this work, the projection scheme proposed by Bourdin [7] has also been applied as filtering technique to prevent mesh dependence and checkerboard problems in the results of TOM. Here, the pseudo-density of a given element of the design domain is a linear function of the design variables associated with their neighborhood. Thus, the following relation is defined to the pseudo-density $\left(d_{e}\right)$ and the design variable $\left(\rho_{i}\right)$ :

$$
d_{e}=f\left(\rho_{i}\right)
$$

where $f$ is a projection function [9].

As expected, the mean compliance minimization [8] will result in very rigid structures, which is undesirable for the flexible structure design because its performance would be very low. As mentioned previously, the difference between two structures illustrated in figure 1 is the perimeter, thus, the definition of a perimeter constraint can be an interesting way to guide the optimization to achieve topologies shown in figure $1 \mathrm{~b}$. To calculate the perimeter of the structure, the Total Variation (TV) concept introduced by Haber et al. [10] is applied. The TV in the domain $\Omega$ for a function $\rho \in L_{l}(\Omega)$, is defined as:

$$
T V(\rho)=\int_{\Omega}|\nabla \rho| d \Omega
$$

The value of TV coincides with the perimeter of $|\Omega|$ when $\rho$ has unit value in $|\Omega|$ and zero outside. In different situations, when the density of the different subdomains varies linearly between the "void" (no material) and solid (presence of material), as made by the SIMP, the definition of equation (2) would represent an approximation of the actual perimeter of the structure. Thus, Zhang \& Duysinx [11] proposes the following definition, in discrete form, to calculate the perimeter $P$ of the topology:

$$
P(\rho)=\sum_{k=1}^{n_{l}} l_{k}\left(d_{i}-d_{j}\right)^{2}
$$

where $n_{l}$ is the total number of interfaces between the elements of the domain and $l_{k}$ denotes the edge length of the interface $k$ between two adjacent elements with pseudo-densities $d_{i}$ and $d_{j}$. For the perimeter calculation of the boundary edges of the domain, the density of the external environment is considered equal to zero.

Therefore, in this work, the following optimization formulation that uses a perimeter constraint are proposed for the design of flexible structures:

$$
\begin{array}{rll}
\underset{d}{\text { Minimize }} & L=\mathbf{u}^{T} \mathbf{K} \mathbf{u} & \\
\text { S.t. } & \mathbf{K} \mathbf{u}=\mathbf{F} & \text { (equilibrium equation) } \\
& \sum_{i=1}^{n e} \rho_{i} V_{i} \leq V^{*} & \text { (volume constraint) } \\
& \sum_{k=1}^{n_{i}} l_{k}\left(d_{i}-d_{j}\right)^{2} \geq P_{\min } & \text { (perimeter constraint) } \\
& \mathbf{d}=f(\boldsymbol{\rho}) & \\
& 0 \leq \boldsymbol{\rho} \leq 1 &
\end{array}
$$


where the superscript ${ }^{\mathrm{T}}$ denotes the transpose vector, $L$ is the mean compliance, $\mathbf{K}$ is the stiffness matrix, and $\mathbf{u}$ is the displacement vector yielded by applying the load vector $\mathbf{F}$, ne is the total number of elements in the design domain, $V_{i}$ is volume of the individual elements, and $P_{\min }$ is the minimum value for the perimeter of the topology.

Traditionally, the perimeter constraint is used as an alternative to the projection scheme, for limiting the occurrence of the checkerboard pattern and, thereby, ensures the existence of the solution [12]. For this, an upper limit $P_{\max }$ is imposed on TV defined by equation (2), i.e., $\operatorname{TV}(\rho)<P_{\max }$. However, in this work the perimeter constraint equation is defined as $\operatorname{TV}(\rho)>P_{\max }$ to guide the optimization for results with high perimeter. To avoid the appearance of checkerboard patterns in the optimization result, this perimeter constraint is used together with the projection technique.

Thus, by using the proposed formulation (4), our goal is to show that a perimeter constraint can make the difference for obtaining higher flexible structures even by employing the classical topology optimization formulation, which minimizes the mean compliance subjected to volume constraint.

\section{Sensitivity Analysis}

To perform the topology optimization, calculation of gradients of the objective functions and constraints are needed. Here, these gradients are calculated analytically by using the adjoint method. This sensitivity analysis is straightforward and it will be briefly described. The gradient of the mean compliance in relation to the pseudo-density $\left(d_{e}\right)$ is given by:

$$
\frac{\partial L}{\partial d_{e}}=\frac{\partial\left(\mathbf{u}^{T} \mathbf{K} \mathbf{u}\right)}{\partial d_{e}}=-\mathbf{u}^{T} \frac{\partial \mathbf{K}}{\partial d_{e}} \mathbf{u}
$$

However, a sensitivity analysis in relation to the design variable $\left(\rho_{i}\right)$ is also needed, which is calculated by using the equation (1) as following:

$$
\frac{\partial L}{\partial \rho_{i}}=\sum_{i \in S_{e}} \frac{\partial L}{\partial d_{e}} \frac{\partial d_{e}}{\partial \rho_{i}}, \quad \text { where } \frac{\partial d_{e}}{\partial \rho_{i}}=\frac{w\left(\overline{\mathbf{x}}^{i}-\overline{\mathbf{x}}^{e}\right)}{\sum_{i \in S_{e}} w\left(\overline{\mathbf{x}}^{i}-\overline{\mathbf{x}}^{e}\right)}
$$

Analogously, from equation (6) the gradient of the perimeter constraint is calculated by:

$$
\frac{\partial P}{\partial \rho_{i}}=\sum_{i \in S_{e}} \frac{\partial P}{\partial d_{e}} \frac{\partial d_{e}}{\partial \rho_{i}}, \quad \text { where } \frac{\partial P}{\partial d_{e}}=2 \sum_{k=1}^{n_{l}} l_{k}\left|d_{e}-d_{j}\right| .
$$

\section{Numerical Implementation of the Topology Optimization Algorithm}

The code of the topology optimization algorithm is implemented in MATLAB by using the COMSOL software as the finite element solver. At each iteration of the topology optimization algorithm, a structural analysis is carried out by using Finite Element Method (FEM), to account for the calculation of the output displacement of the structure. In this work, the design domain is discretized by using a linear isoparametric 4-node Mindlin-Reissner-based plate with 6 degrees of freedom per node [13].

The gradients of the objective function and constraints are calculated. They are used as input for the optimization problem, which is solved by using the Method of Moving Asymptotes (MMA) [14]. The optimizer generates a new set of design variables after each iteration and the optimization continues until convergence is achieved for the objective function.

\section{Results}

In this section a well-known two-dimensional (2D) plane string case has been carried out to demonstrate the potential of the topology optimization formulation for solving design of highly flexible structures. Figure 2a shows the design domain, which is discretized by using a 120x120 finite element mesh. The left side is full constrained, and a unitary load (F) is applied at the center of the right side of the domain $(\Omega)$. To highlight the feasibility of including perimeter control for increasing the level of flexibility, some results, shown in figure $2 b$, have been carried out by using the modified classical formulation given by equation (4). A volume fraction $V^{*}=10 \%$ and the projection radius $r_{\min }=2$ are applied for all results obtained in this case. For easy comparison, various minimum values for $P_{\min }$ are applied to the perimeter of the structure, which is gradually increased from 0 up to 1000 (see figure $2 b$ ).

The mean compliance value $(L)$ is shown in the upper left corner of each topology result presented in figure $2 \mathrm{~b}$. As expected, the first result shown in figure $2 \mathrm{~b}\left(P_{\min }=0\right)$ is a tapered column structure, which has low flexibility $(L=13)$ and perimeter $P=186$. The simple inclusion of the perimeter constraint $P_{\min }=200$ has enabled an increase of almost 10 times in the magnitude of the structural flexibility, as observed in the second result of figure $2 \mathrm{~b}$. Observing all the results, it is clear that there is a strong relationship between increased perimeter and flexibility of the structure. Moreover, it is noticed from a certain point $\left(P_{\min }=1000\right)$ appearance of disconnected structures (islands of material) can occur, which from a point of view of the structural strength has little influence, however contributes significantly to increase the perimeter. These islands of materials must be removed by a post-processing step. The appearance of islands can be avoided by adjusting the value of the 
volume constraint and perimeter constraint.

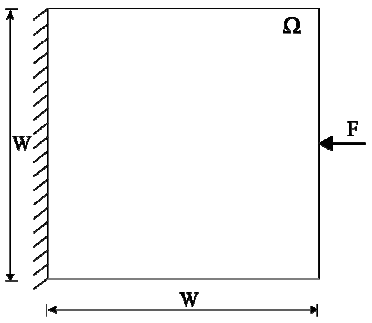

(a)

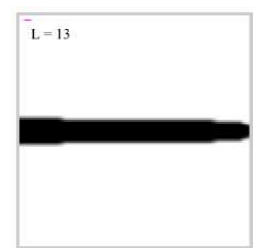

(a) $P_{\min }=0$

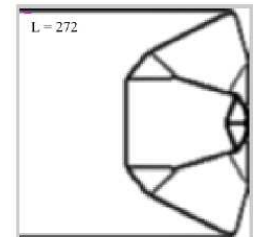

(d) $P_{\min }=600$

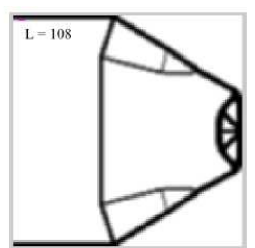

(b) $P_{\min }=200$

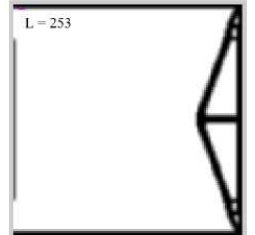

(e) $P_{\text {min }}=800$

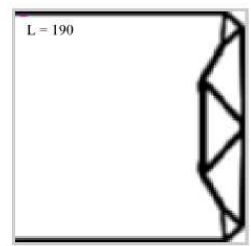

(c) $P_{\min }=400$

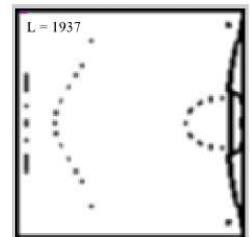

(f) $P_{\min }=1000$

(b)

Figure 2 - (a) Design domain of the topology optimization problem; (b) topology optimization results obtained by including various perimeter constraint values.

\section{Conclusion}

A methodology for the design of high flexible structures by using the Topology Optimization Method is presented and evaluated by considering a 2D plane string design. Thus, a different approach is proposed, in which the optimization problem has been defined for minimizing the mean compliance subjected to volume and perimeter constraints, together with the projection technique. Structures with higher flexibility have been generated through the proposed approach presented in this work.

Despite the relative success of the formulation presented here, this subject needs to be more studied and exhausted. It is essential to propose new formulations for the TOM to solve this kind of problem, which certainly has a wide range of applications beyond that already discussed in this work. As future work, other formulations which allow the design of structures with a specified level of the flexibility should be included and evaluated more carefully in this context.

\section{Acknowledgements}

The authors thank the financial support given by CNPq (grants no. 305782/2012-6 and 304121/2013-4), and FINEP (grant no. 01.09.0395.00). The authors are grateful to Krister Svanberg from KTH Royal Institute of Technology for supplying the MMA-code.

\section{References}

1. D. Qiao, G. Pang, M-K. Mui, and D. Lam, A single-axis low-cost accelerometer fabricated using printed-circuit-board techniques. Electron Device Letters, IEEE, 30 (2009), 1293-1295

2. S. Desrochers, D. Pasini, and J. Angeles, Optimum design of a compliant uniaxial accelerometer. Journal of Mechanical Design, 132 (2010).

3. S. Nishiwaki, M.I. Frecker, S. Min, and N. Kikuchi, Topology optimization of compliant mechanisms using the homogenization method. International Journal for Numerical Methods in Engineering, 42 (1998), 535-559.

4. M-G. Song, N-C. Park, K-S. Park, and Y-P. Park, Design of a leaf spring using a genetic algorithm. IEEE Trans. on Magnetics, 47 (2011), $590-593$.

5. M.P. Bendsøe, Optimal shape design as a material distribution problem. Structural and Multidisciplinary Optimization, 1 (1989), $193-202$.

6. M.P. Bendsøe and O. Sigmund, (2003) Topology Optimization: Theory, Methods and Applications. Springer Verlag.

7. B. Bourdin, Filters in topology optimization. International Journal for Numerical Methods In Engineering, 50 (2001), $2143-2158$.

8. K. Suzuki and N. Kikuchi, A homogenization method for shape and topology optimization. Computer Methods in Applied Mechanics and Engineering, 93 (1991), 291-318.

9. T.E. Bruns and D.A. Tortorelli, Topology optimization of non-linear elastic structures and compliant mechanisms. Computer Methods in Applied Mechanics and Engineering. 190 (2001) 3443-3459.

10. R.B. Haber, C.S. Jog, and M.P. Bendsøe, A new approach to variable-topology shape design using a constraint on perimeter. Structural and Multidisciplinary Optimization, 11 (1996), 1-12.

11. W. Zhang and P. Duysinx, Dual approach using a variant perimeter constraint and efficient sub-iteration scheme for topology optimization. Computers \& Structures, 81 (2003), 2173-2181.

12. L. Ambrosio and G. Buttazzo, An optimal design problem with perimeter penalization. Calculus of Variations and Partial Differential Equations, 1 (1993), 55-69.

13. D. Chapelle and K. J. Bathe, (2003) The Finite Element Analysis of Shells: Fundamentals. Springer.

14. K. Svanberg, The method of moving asymptotes: a new method for structural optimization. International Journal for Numerical Methods in Engineering, 24 (1987), 359-373. 